\title{
Alkylation of 2,4-(1H,3H)-Quinazolinediones with Dialkyl Carbonates Under Microwave Irradiations
}

\section{Ignacio Alfredo Rivero ${ }^{1,2, *}$, Leticia Guerrero ${ }^{1}$, Karla Alejandra Espinoza ${ }^{3}$, Martha Cecilia Meza $^{1}$ and Jesús Ramón Rodríguez ${ }^{3}$}

1 Centro de Graduados e Investigación en Química, Instituto Tecnológico de Tijuana. C.P. 1166. Tijuana, B.C. 22000, Mexico

2 Instituto Nacional de Investigaciones Nucleares, Departamento de Química. Carretera México Toluca S/N, La Marquesa, Ocoyoacac, Mexico, D.F. C.P. 52750

3 Facultad de Química, Universidad Autónoma de Baja California, Calzada Tecnológico \#14418, Mesa de Otay. Tijuana, B.C, Mexico, C.P. 22390

* Author to whom correspondence should be addressed; E-mail: irivero@tectijuana.mx; Tel.: +52664-6233772; Fax: +52-664-6234043

Received: 16 March 2009; in revised form: 6 April 2009 / Accepted: 9 April 2009 / Published: 20 May 2009

\begin{abstract}
Alkylation is a very important chemical reaction which modifies the biological properties of drugs. Quinazolinedione derivatives are of considerable interest due to their wide array of pharmacological properties. We now report application of a practical alkylation procedure to several quinazolinediones, including pelanserine (5f), which shows antihypertensive properties, 1-methyl-3-(2'-phenylethyl)-1H,3H-quinazoline-2,4-dione (1ab) and 1-methyl-3-[2'-(4'-methoxyphenyl)ethyl]-1H,3H-quinazoline-2,4-dione (1ae), which had been isolated from natural sources. The alkylation was optimized using dimethyl and diethyl carbonates under microwave irradiations.
\end{abstract}

Keywords: alkylation; quinazoline-2,4-dione; antihypertensive activity; microwaves 


\section{Introduction}

In general alkylation involves substitution of a hydrogen atom by an alkyl group and is a popular and fundamental process in organic synthesis. Several functional groups as $\alpha$-carbon [1], alcohols [2], amines [3], carboxylic acids [4] and amides-NH [5] are protected by alkylation reactions. These modifications change the physic chemical and biological properties of such compounds. Our group has been working on the synthesis of quinazolinone and quinazolinedione derivatives, which are of considerable interest because of their wide array of pharmacological properties [6-20]. We have synthesized heterocycles containing the quinazoline-2,4-dione backbone, which are known to exhibit potential anti-hypertensive properties [21-25]. We have described the synthesis of pelanserine (5f), a potent anti-hypertensive agent [26], and a several quinazoline-2,4-diones with amino acids and dipeptide, which when tested showed mild to no antihypertensive properties [27].

Recently, we synthesized two alkaloids containing the quinazoline-2,4-dione ring skeleton 1-methyl-3-(2'-phenylethyl)-1H,3H-quinazoline-2,4-dione (1ab) and 1-methyl-3-[2'-(4'-methoxyphenyl)ethyl]-1H,3H-quinazoline-2,4-dione (1ae) - which have been isolated from the seed husks of Zanthoxylum arborescens [28]. Alkylation reactions were performed with methyl iodide and TMG as a base, but the reagents are expensive $\underline{a b d}$ toxic and take about one hour at $55^{\circ} \mathrm{C}$ to complete the reaction [29]. Herein, we propose a novel methodology for the alkylation using inexpensive dimethyl and diethyl carbonates which are very stable liquids, non-reactive under normal conditions. The reactions were assisted and optimized by microwaves, taking only a few minutes to complete. This efficient process was applied to a quinazolinedione library to improve the methodology without toxic reagents.

Figure 1. Examples of quinazoline-2,4- diones: Pelanserine (5f) a potent anti-hypertensive agent, and two natural products $[5(\mathbf{b}, \mathbf{e})]$.

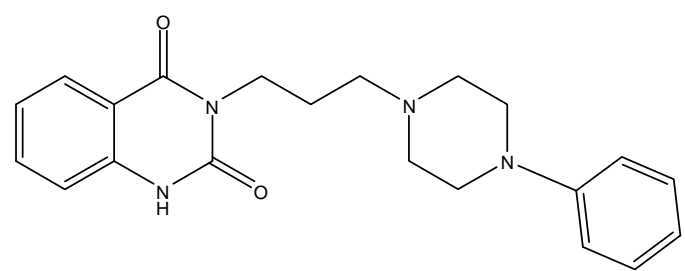

$5 \mathbf{f}$

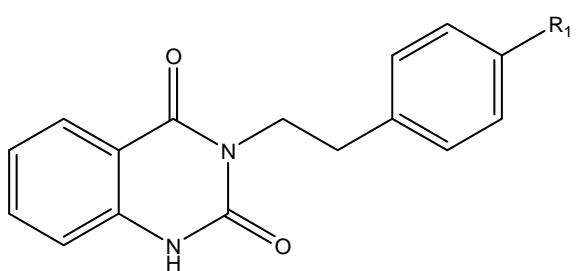

$5 \mathbf{b}\left(\mathrm{R}_{1}=\mathrm{H}\right), \mathbf{5 e}\left(\mathrm{R}_{1}=\mathrm{OCH}_{3}\right)$

\section{Results and Discussion}

Alkylation of $\mathrm{NH}$-containing heteroaromatic compounds is an important transformation that regularly employs toxic and hazardous reagents such as methyl iodide [29] or dimethyl sulfate [30]. Dialkyl carbonates are an attractive alternative as alkylating reagents for $\mathrm{NH}$-containing heteroaromatic compounds. Quinazoline-2,4-diones $\mathbf{5}(\mathbf{a}, \mathbf{b}, \mathbf{c}, \mathbf{d}, \mathbf{e}, \mathbf{f})$ were thus prepared using our methodology [26]. Initially, we prepared the ortho-aminobenzamides from the reactions of isatoic anhydride with amines and the cyclization was carried out with bis(trichloride methyl)carbonate (BTC, triphosgene). Finally, the alkylation with dimethyl carbonate was optimized under microwave irradiation and the conditions were fixed at $200 \mathrm{~W}, 130{ }^{\circ} \mathrm{C}$, for 15 minutes, using $\mathrm{K}_{2} \mathrm{CO}_{3}$ as base 
(Scheme 1). The reaction was filtered, to get a $>94 \%$ yield. The alkylation with diethyl carbonate was similar, in this case it was necessary to increase the temperature to $160{ }^{\circ} \mathrm{C}$ to obtain the ethylquinazoline-2,4-diones in a $>92 \%$ yield. Ethylation products had to be purified by column chromatography on silica gel to remove the excess of diethyl carbonate. By applying microwave irradiation further rate enhancements were accomplished.

Scheme 1. Steps to obtain to the N-methyl quinazoline-2,4-diones.<smiles>O=c1[nH]c2ccccc2c(=O)o1</smiles>

(2)
(3)<smiles>[R19]C1CC1C(=O)CO</smiles>

iii)<smiles>[R]n1c(=O)c2ccccc2n([R])c1=O</smiles>

(1)<smiles>[R1]NC(=O)c1ccccc1N</smiles>

(4)<smiles>[R1]n1c(=O)[nH]c2ccccc2c1=O</smiles>

(5)

$\mathrm{R}^{1}$ : a) $\mathrm{PhCH}_{2} \quad$ b) $2-\mathrm{CH}_{3} \mathrm{OPhCH}_{2} \mathrm{CH}_{2}$ c) $3-\mathrm{CH}_{3} \mathrm{OPhCH}_{2} \mathrm{CH}_{2}$

d) $4-\mathrm{CH}_{3} \mathrm{OPhCH}_{2} \mathrm{CH}_{2}$ e) $\mathrm{PhCH}_{2} \mathrm{CH}_{2}$ f) $\mathrm{PhN}\left(\mathrm{CH}_{2}\right)_{4} \mathrm{NCH}_{2} \mathrm{CH}_{2} \mathrm{CH}_{2}$ $\mathrm{R}^{2}$ : a) $\mathrm{CH}_{3}$ b) $\mathrm{CH}_{3} \mathrm{CH}_{2}$
i) $\mathrm{DMF} / 60^{\circ} \mathrm{C}$
ii) $\mathrm{BTC} / \mathrm{CH}_{2} \mathrm{Cl}_{2}$
iii) $\left(\mathrm{R}_{2} \mathrm{CO}\right)_{2} \mathrm{O} / \mathrm{K}_{2} \mathrm{CO}_{3}$

The new methodology has the advantages of rapid reaction times, ease of operation and purification and the use of readily available reagents, and the avoidance of toxic alkylating reagents. In this work methyl and ethyl quinazoline-2,4-dione libraries were prepared, which are detailed in Table 1. The yields obtained with the optimized method were excellent, with conversions of $92-98 \%$. Therefore, this is a very efficient method, with easy purification of products, since no by-products were observed.<smiles>[R]n1c(=O)c2ccccc2n([R])c1=O</smiles>

(1) 
Table 1. Alkylated quinazoline-2,4-diones.

\begin{tabular}{|c|c|c|c|}
\hline Entries & $\mathbf{R}^{\mathbf{1}}$ & $\mathbf{R}^{\mathbf{2}}$ & \% Yield \\
\hline 1aa & $\mathrm{PhCH}_{2}$ & $\mathrm{CH}_{3}$ & 94 \\
1ab & $\mathrm{PhCH}_{2} \mathrm{CH}_{2}$ & $\mathrm{CH}_{3}$ & 98 \\
1ac & $2-\mathrm{CH}_{3} \mathrm{OPhCH}_{2} \mathrm{CH}_{2}$ & $\mathrm{CH}_{3}$ & 95 \\
1ad & $3-\mathrm{CH}_{3} \mathrm{OPhCH}_{2} \mathrm{CH}_{2}$ & $\mathrm{CH}_{3}$ & 96 \\
1ae & $4-\mathrm{CH}_{3} \mathrm{OPhCH}_{2} \mathrm{CH}_{2}$ & $\mathrm{CH}_{3}$ & 98 \\
1af & $\mathrm{PhN}\left(\mathrm{CH}_{2}\right)_{4} \mathrm{NCH}_{2} \mathrm{CH}_{2} \mathrm{CH}_{2}$ & $\mathrm{CH}_{3}$ & 93 \\
1ba & $\mathrm{PhCH}_{2}$ & $\mathrm{CH}_{3} \mathrm{CH}_{2}$ & 96 \\
1bb & $\mathrm{PhCH}_{2} \mathrm{CH}_{2}$ & $\mathrm{CH}_{3} \mathrm{CH}_{2}$ & 96 \\
1bc & $2-\mathrm{CH}_{3} \mathrm{OPhCH}_{2} \mathrm{CH}_{2}$ & $\mathrm{CH}_{3} \mathrm{CH}_{2}$ & 95 \\
1bd & $3-\mathrm{CH}_{3} \mathrm{OPhCH}_{2} \mathrm{CH}_{2}$ & $\mathrm{CH}_{3} \mathrm{CH}_{2}$ & 94 \\
1be & $4-\mathrm{CH}_{3} \mathrm{OPhCH}_{2} \mathrm{CH}_{2}$ & $\mathrm{CH}_{3} \mathrm{CH}_{2}$ & 97 \\
1bf & $\mathrm{PhN}\left(\mathrm{CH}_{2}\right)_{4} \mathrm{NCH}_{2} \mathrm{CH}_{2} \mathrm{CH}_{2}$ & $\mathrm{CH}_{3} \mathrm{CH}_{2}$ & 92 \\
\hline
\end{tabular}

\section{Conclusions}

We have developed a simple method to methyl or ethyl alkylation of amide- $N H$ functions with dialkyl carbonates, which were assisted by microwave irradiation. We used several quinazoline-2,4diones which were previously synthesized by our group for biological evaluation as potential antihypertensive agents. We have proven that this method is very fast, clean, with almost complete conversion, using stable reagents avoiding possible contamination. We are currently exploring this reaction without solvent, as a green chemistry process. The amounts of $\mathrm{K}_{2} \mathrm{CO}_{3}$ are important which were established at three equivalents, thus working in a more efficient way. After several experiments, optimum conditions were determined. The basic backbone provides a source for introduction of different heterocyclic extension on the amide $-\mathrm{NH}$, in order to diversify the quinazoline-2,4-dione structural system.

\section{Experimental}

\section{General}

Melting points were measured on an Electrothermal 88629 apparatus and are uncorrected. Infrared (IR) spectra were recorded on a Perkin Elmer FT-IR 1600 spectrometer. ${ }^{1} \mathrm{H}-\mathrm{NMR}$ and ${ }^{13} \mathrm{C}-\mathrm{NMR}$ spectra were recorded at $200 \mathrm{MHz}$ and $50.289 \mathrm{MHz}$, respectively, on a Varian Mercury 200 spectrometer in $\mathrm{CDCl}_{3}$ with TMS as internal standard. Mass spectra were obtained on an Agilent 1100 series LC/MSD Trap, SL Spectrometer by electrospray insertion. Microwave equipment was a selftuning single mode CEM Discover ${ }^{\mathrm{TM}}$ Focused Synthesizer.

\section{General Method for Methylation of Quinazoline-2,4-diones 4}

1-Methyl-3-(2'-phenylethyl)-1H,3H-quinazoline-2,4-dione (1ab). Dimethyl carbonate (1.25 mL) was added to a solution of 3-phenylethyl-1H-quinazoline-2,4-dione (5b, 0.125g, $0.38 \mathrm{mmol})$ in DMF 
(1.25 mL) and $\mathrm{K}_{2} \mathrm{CO}_{3}$ (3 equiv) as base. The mixture was placed in a microwave reactor vessel $\left(10 \mathrm{~mL}\right.$ ) and heated at $130{ }^{\circ} \mathrm{C}$ for 15 minutes and cooled to RT, then diluted with $\mathrm{CH}_{2} \mathrm{Cl}_{2}$ and $\mathrm{H}_{2} \mathrm{O}$. The aqueous layer was removed, and the organic layer was washed with $\mathrm{H}_{2} \mathrm{O}$, twice with $2 \mathrm{M} \mathrm{HCl}$ or $10 \%$ aqueous citric acid, twice with saturated aqueous $\mathrm{NaHCO}_{3}$, and twice with $\mathrm{H}_{2} \mathrm{O}$. The organic layer was dried over anhydrous $\mathrm{Na}_{2} \mathrm{SO}_{4}$, filtered, and concentrated under vacuum to afford $\mathbf{1} \mathbf{a b}$ as a white solid. Yield $>98 \%$; $\mathrm{mp} 99-101^{\circ} \mathrm{C}$ (Lit. [25], mp. 100-102 ${ }^{\circ} \mathrm{C}$ ); IR (KBr): 3042, 2929, 1701, 1654, 1610, $1481 \mathrm{~cm}^{-1}$; ${ }^{1} \mathrm{H}-\mathrm{NMR}$ : $\delta 8.23\left(\mathrm{dd}, 1 \mathrm{H}, J_{I}=1.7, J_{2}=7,8 \mathrm{~Hz}, \mathrm{Ar}-\mathrm{H}\right), 7.65\left(\mathrm{ddd}, 1 \mathrm{H}, J_{I}=1,7, J_{2}=7,3\right.$, $\left.J_{3}=8,4 \mathrm{~Hz}, \mathrm{Ar}-\mathrm{H}\right), 7.34-7.16(\mathrm{~m}, 7 \mathrm{H}, \mathrm{Ar}-\mathrm{H}), 4.29$ (ddd, $\left.J_{I}=5.6, J_{2}=J_{3}=7.8 \mathrm{~Hz}, 2 \mathrm{H}, \mathrm{N}-\mathrm{CH}_{2}\right), 3.57(\mathrm{~s}$, $3 \mathrm{H}, \mathrm{N}-\mathrm{CH}_{3}$ ), $2.96\left(\mathrm{ddd}, \mathrm{J}_{1}=5.2, \mathrm{~J}_{2}=\mathrm{J}_{3}=8,0 \mathrm{~Hz}, \mathrm{Ar}-\mathrm{CH}_{2}\right.$ ) ppm; ${ }^{13} \mathrm{C}-\mathrm{NMR}: \delta 161.3,150.5,140.2,138.3$, $134.8,129.9$, 128.7, 128.6, 128.2, 126.2, 122.7, 120.9, 113.3, 43.3, 33.9, 30.6 ppm; ESI-MS (m/e): $280.1[\mathrm{M}+\mathrm{H}]^{+}$.

The following compounds were prepared in similar fashion:

1-Methyl-3-[2'-(2'-methoxyphenyl)ethyl]-lH,3H-quinazoline-2,4-dione (1ac). White solid. Yield >95\%; mp 155-163을 IR (KBr): 2943, 1703, 1651, 1608, 1484, 1243, $1028 \mathrm{~cm}^{-1}$; ${ }^{1} \mathrm{H}-\mathrm{NMR}$ : $\delta 8.21$ $\left(\mathrm{dd}, 1 \mathrm{H}, J_{I}=1.6, J_{2}=7.9 \mathrm{~Hz}, \mathrm{Ar}-\mathrm{H}\right), 7.66\left(\mathrm{ddd}, 1 \mathrm{H}, J_{l}=1.7, J_{2}=7.3, J_{3}=8.5 \mathrm{~Hz}, 1 \mathrm{H}, \mathrm{Ar}-\mathrm{H}\right), 7.28-7.16$ (m, 5H, Ar-H), 6.85(ddd, $\left.1 \mathrm{H}, J_{l}=1.0, J_{2}=J_{3}=8.2 \mathrm{~Hz}, \mathrm{Ar}-\mathrm{H}\right), 4.33\left(\mathrm{ddd}, 2 \mathrm{H}, J_{I}=5.6, J_{2}=J_{3}=7.4 \mathrm{~Hz}, \mathrm{Ar}-\right.$ $\left.\mathrm{CH}_{2}\right), 3.81\left(\mathrm{~s}, 3 \mathrm{H}, \mathrm{O}-\mathrm{CH}_{3}\right), 3.58\left(\mathrm{~s}, 3 \mathrm{H}, \mathrm{N}-\mathrm{CH}_{3}\right), 3.02$ (ddd, $2 \mathrm{H}, J_{l}=5.8, J_{2}=J_{3}=7.4 \mathrm{~Hz}, \mathrm{Ar}-\mathrm{CH}_{2}$ ) ppm; ${ }^{13}$ C-NMR: $\delta$ 161.3, 157.8, 140.2, 134.9, 130.6, 128.6, 127.7, 127.1, 122.8, 120.4, 113.4, 110.2, 55.3, 41.8, 30.6, 28.7 ppm; ESI-MS (m/e): $310.9[\mathrm{M}+\mathrm{H}]^{+} ; 332[\mathrm{M}+\mathrm{Na}]^{+}$.

1-Methyl-3-[2'-(3'-methoxyphenyl)ethyl]-lH,3H-quinazoline-2,4-dione (1ad). White solid. Yield 96\%; mp 131-133 ${ }^{\circ} \mathrm{C}$; IR (KBr): 2945, 2833, 1699, 1656, $1604 \mathrm{~cm}^{-1}$; ${ }^{1} \mathrm{H}-\mathrm{NMR}: \delta 8.23$ (dd, $1 \mathrm{H}, J_{l}=1.7$, $\left.J_{2}=7.9 \mathrm{~Hz}, \mathrm{Ar}-\mathrm{H}\right), 7.63\left(\mathrm{ddd}, 1 \mathrm{H}, J_{1}=1.6, J_{2}=7.2, J_{3}=8.4 \mathrm{~Hz}, \operatorname{Ar}-\mathrm{H}\right), 7.25-7.15(\mathrm{~m}, 4 \mathrm{H}, \operatorname{Ar}-\mathrm{H})$, $6.78\left(\mathrm{ddd}, 1 \mathrm{H}, J_{I}=1.0, J_{2}=2.5, J_{3}=8.2 \mathrm{~Hz}, \mathrm{Ar}-\mathrm{H}\right), 4.30\left(\mathrm{ddd}, 2 \mathrm{H}, J_{I}=5.4, J_{2}=J_{3}=7.6 \mathrm{~Hz}, \mathrm{~N}-\mathrm{CH}_{2}\right)$, $3.79\left(\mathrm{~s}, 3 \mathrm{H}, \mathrm{O}-\mathrm{CH}_{3}\right), 3.55\left(\mathrm{~s}, 3 \mathrm{H}, \mathrm{N}-\mathrm{CH}_{3}\right), 2.98\left(\mathrm{ddd}, 2 \mathrm{H}, J_{l}=5.1, J_{2}=J_{3}=7.6 \mathrm{~Hz}, \mathrm{Ar}-\mathrm{CH}_{2}\right)$ ppm; ${ }^{13} \mathrm{C}-\mathrm{NMR}: \delta$ 162.5, 159.9, 148.5, 140.5, 132.8, 132.4, 129.7, 127.0, 126.4, 122.7, 117.4, 116.8, 116.3, 112.1, 55.1, 40.7, 35.7 ppm; ESI-MS (m/e): $332.9[\mathrm{M}+\mathrm{Na}]^{+}$

1-Methyl-3-[2'-(4'-methoxyphenyl)ethyl]-lH,3H-quinazoline-2,4-dione (1ae). White solid. Yield $>98 \%$; mp. 134- $136^{\circ} \mathrm{C}$. (Lit. [25] mp. 133-134 ${ }^{\circ} \mathrm{C}$ ); IR (KBr): 3301, 2928, 1700, 1647, 1600, 1400, $1261 \mathrm{~cm}^{-1} ;{ }^{1} \mathrm{H}-\mathrm{NMR}$ : $\delta 8.21\left(\mathrm{dd}, 1 \mathrm{H}, J_{l}=1.6, J_{2}=7.8 \mathrm{~Hz}, \mathrm{Ar}-\mathrm{H}\right), 7.69\left(\mathrm{ddd}, 1 \mathrm{H}, J_{I}=1.7, J_{2}=7.4, J_{3}=8.5\right.$ $\mathrm{Hz}, \mathrm{Ar}-\mathrm{H}), 7.30-7.18(\mathrm{~m}, 6 \mathrm{H}, \mathrm{Ar}-\mathrm{H}), 6.80\left(\mathrm{ddd}, 1 \mathrm{H}, J_{l}=1.0, J_{2}=2.5, J_{3}=8.2 \mathrm{~Hz}, \mathrm{Ar}-\mathrm{H}\right), 4.26$ (ddd, 2H, $\left.J_{I}=5.2, J_{2}=J_{3}=7.8 \mathrm{~Hz}, \mathrm{~N}-\mathrm{CH}_{2}\right), 3.80\left(\mathrm{~s}, 3 \mathrm{H}, \mathrm{O}-\mathrm{CH}_{3}\right), 3.59\left(\mathrm{~s}, 3 \mathrm{H}, \mathrm{N}-\mathrm{CH}_{3}\right), 2.95\left(\mathrm{ddd}, 2 \mathrm{H}, J_{I}=5.2\right.$, $J_{2}=J_{3}=7.8 \mathrm{~Hz}, \mathrm{Ar}-\mathrm{CH}_{2}$ ) ppm; ${ }^{13} \mathrm{C}-\mathrm{NMR}: \delta 161.8,158.2,140.5,130.1,129.2,124.2,114.0,113.6,55.5$, 43.7, 33.4, 31.0 ppm; ESI-MS (m/e): $332.9[\mathrm{M}+\mathrm{Na}]^{+}$.

1-Methyl-3-(benzyl)-1H,3H-quinazoline-2,4-dione (1aa). White solid. Yield 94\%; mp.103-106 ${ }^{\circ}$; IR (KBr): 3416, 2918, 1700, 1652, 1604, 1480, 1266, $\mathrm{cm}^{-1}$; ${ }^{1} \mathrm{H}-\mathrm{NMR}: \delta 8.22$ (dd, $1 \mathrm{H}, J_{I}=1.6, J_{2}=7.9 \mathrm{~Hz}$, Ar-H), 7.64 (ddd, $1 \mathrm{H}, J_{I}=1.6, J_{2}=7.3, J_{3}=8.5 \mathrm{~Hz}, \mathrm{Ar}-\mathrm{H}$ ), 7.52 (dd, 2H, $\left.J_{I}=1.7, J_{2}=7.8 \mathrm{~Hz}, \mathrm{Ar}-\mathrm{H}\right), 7.34$ - 
$7.13(\mathrm{~m}, 5 \mathrm{H}, \mathrm{Ar}-\mathrm{H}), 5.27$ (s, 2H, N-CH$), 3.57$ (s, 3H, N-CH 3$)$ ppm; ${ }^{13} \mathrm{C}-\mathrm{NMR}: \delta 161.7,150.9,140.9$, 137.0, 135.1, 129.7, 129.0, 128.3, 127.5, 122.9, 115.5, 113.5, 44.9, 30.7 ppm; ESI-MS (m/e) 288.9 $[\mathrm{M}+\mathrm{Na}]^{+}$.

1Methyl-3-(3-(4-phenylpiperazin-1-yl)propyl)-1H,3H-quinazoline-2,4-dione (1af). White solid. Yield $>93 \%$; IR (KBr): 3018, 2932, 2880, 2803, 1695, 1647, 1604, 1223, $1110 \mathrm{~cm}^{-1} .{ }^{1} \mathrm{H}-\mathrm{NMR}: \delta 8.18$ (dd, $\left.1 \mathrm{H}, J_{1}=1.6, J_{2}=7.9 \mathrm{~Hz}, \mathrm{Ar}-\mathrm{H}\right), 7.62\left(\mathrm{ddd}, 1 \mathrm{H}, J_{l}=1.6, J_{2}=7.4, J_{3}=8.4 \mathrm{~Hz}, \mathrm{Ar}-\mathrm{H}\right), 7.25-7.11(\mathrm{~m}, 3 \mathrm{H}, \mathrm{Ar}-$ $\mathrm{H})$, 6.88-6.75 (m, 3H, Ar-H), $4.16\left(\mathrm{~m}, 2 \mathrm{H}, \mathrm{N}-\mathrm{CH}_{2}\right), 3.58\left(\mathrm{~s}, 1 \mathrm{H}, \mathrm{N}-\mathrm{CH}_{3}\right), 3.10\left(\mathrm{~m}, 4 \mathrm{H}, \mathrm{N}-\mathrm{CH}_{2}\right)$, $2.55\left(\mathrm{~m}, 6 \mathrm{H}, \mathrm{N}-\mathrm{CH}_{2}\right), 1.95\left(\mathrm{dd}, 3 \mathrm{H}, J_{l}=7.1, J_{2}=14.3 \mathrm{~Hz}, \mathrm{CH}_{2}\right) \mathrm{ppm} ;{ }^{13} \mathrm{C}-\mathrm{NMR}: \delta 161.8,151.3,148.8$, $140.5,135.0$, 129.1, 128.9, 128.8, 122.8, 119.5, 115.9, 112.8, 56.1, 55.2, 53.0, 49.1, 40.5, 30.6, 24.8 ppm; ESI-MS (m/e): $392.2[\mathrm{M}+\mathrm{H}]^{+}$.

\section{General Method for Ethylation of 2,4 Quinazoline-2,4-diones 4}

1-Ethyl-3-(2'-phenylethyl)-1H,3H-quinazoline-2,4-dione (1bb). Diethyl carbonate (1.25 mL), was added to a solution of 3-phenylethyl-1-H-quinazoline-2,4-dione (5b) $(0.125 \mathrm{~g}, 0.38 \mathrm{mmol})$ in DMF $(1.25 \mathrm{~mL})$ and $\mathrm{K}_{2} \mathrm{CO}_{3}$ (3 equiv) as base. The mixture was placed in a microwave reactor vessel $(10 \mathrm{~mL})$ and heated at $160{ }^{\circ} \mathrm{C}$ for 15 minutes and cooled to RT, then diluted with $\mathrm{CH}_{2} \mathrm{Cl}_{2}$ and washed with $\mathrm{NaCl}$ to remove DMF. The organic layer was dried over anhydrous $\mathrm{Na}_{2} \mathrm{SO}_{4}$, filtered, and concentrated under vacuum. The crude was purified by silica gel column chromatography with silica using gel first hexane $(60 \mathrm{~mL})$ and then EtOAc $(60 \mathrm{~mL})$ to give $\mathbf{1 b c}$ as a yellow viscous liquid. Yield $>96 \%$; IR (NaCl): 3033, 2929, 1705, 1657, 1609, 1483, 1402, $1229 \mathrm{~cm}^{-1}$; ${ }^{1} \mathrm{H}-\mathrm{NMR}: \delta 8.25$ (dd, $1 \mathrm{H}$, $\left.J_{I}=1.7, J_{2}=7.8 \mathrm{~Hz}, \mathrm{Ar}-\mathrm{H}\right), 7.68\left(\mathrm{ddd}, 1 \mathrm{H}, J_{I}=1.7, J_{2}=7.3, J_{3}=8.4 \mathrm{~Hz}, \mathrm{Ar}-\mathrm{H}\right), 7.34-7.20(\mathrm{~m}, 7 \mathrm{H}, \mathrm{Ar}-\mathrm{H})$, $4.31\left(\mathrm{ddd}, J_{I}=5.6, J_{2}=J_{3}=7.8 \mathrm{~Hz}, 2 \mathrm{H}, \mathrm{N}-\mathrm{CH}_{2}\right), 4.20\left(\mathrm{q}, 2 \mathrm{H}, J=7.1 \mathrm{~Hz},-\mathrm{CH}_{2}\right), 2.98\left(\mathrm{ddd}, 2 \mathrm{H}, J_{l}=5.2\right.$, $\left.J_{2}=J_{3}=8.0 \mathrm{~Hz}, \mathrm{Ar}-\mathrm{CH}_{2}\right), 1.34\left(\mathrm{t}, 3 \mathrm{H}, J=7.1 \mathrm{~Hz},-\mathrm{CH}_{3}\right) \mathrm{ppm} ;{ }^{13} \mathrm{C}-\mathrm{NMR}: \delta 161.6,150.4,139.5,138.6$, $135.0,129.1,129.0,128.4,126.4,122.7,115.8,113.3,43.2,38.7,34.0,12.5$ ppm; ESI-MS (m/e): $294.1[\mathrm{M}+\mathrm{H}]^{+}$.

The following compounds were prepared in similar fashion:

1-Ethyl-3-(benzyl)-1H,3H-quinazoline-2,4-dione (1ba). White solid. Yield $>96 \%$; mp. 103-105 ${ }^{\circ} \mathrm{C}$; IR (NaCl): 2974, 2922, 1701, 1657, 1605, $1483 \mathrm{~cm}^{-1}$; ${ }^{1} \mathrm{H}-\mathrm{NMR}: \delta 8.26$ (dd, $1 \mathrm{H}, J_{l}=1.7, J_{2}=7.8 \mathrm{~Hz}, \mathrm{Ar}-\mathrm{H}$ ), $7.66\left(\mathrm{ddd}, 1 \mathrm{H}, J_{l}=1.7, J_{2}=7.3, J_{3}=8.4 \mathrm{~Hz}, \mathrm{Ar}-\mathrm{H}\right), 7.52\left(\mathrm{dd}, 2 \mathrm{H}, J_{l}=1.8, J_{2}=7.9 \mathrm{~Hz}, \mathrm{Ar}-\mathrm{H}\right), 7.35-7.18$ (m, 5H, Ar-H), $5.28\left(\mathrm{~s}, 2 \mathrm{H}, \mathrm{N}-\mathrm{CH}_{2}\right), 4.20\left(\mathrm{q}, 2 \mathrm{H}, J=7.1 \mathrm{~Hz},-\mathrm{CH}_{2}\right), 1.34$ (t, 3H, J=7.1 Hz, - $\left.\mathrm{CH}_{3}\right) \mathrm{ppm}$; ${ }^{13}$ C-NMR: $\delta 161.4,150.3,139.6,137.0,135.1,129.3,129.0,128.4,127.5,122.7,120.2,113.3,44.9$, 38.8, 12.5 ppm; ESI-MS (m/e): 280.1[M+ H] $]^{+}$.

1-Ethyl-3-[2'-(2'-methoxyphenyl)ethyl]-lH,3H-quinazoline-2,4-dione (1bc). White solid. Yield >95\%; mp. 128-130 ${ }^{\circ}$; IR (NaCl): 2915, 2848, 1701, 1657, 1605, 1483, $1240 \mathrm{~cm}^{-1}$; ${ }^{1} \mathrm{H}-\mathrm{NMR}: \delta 8.24(\mathrm{dd}, 1 \mathrm{H}$, $\left.J_{I}=1.7, J_{2}=7.8 \mathrm{~Hz}, \mathrm{Ar}-\mathrm{H}\right), 7.66$ (ddd, $\left.1 \mathrm{H}, J_{l}=1.7, J_{2}=7.3, J_{3}=8.4 \mathrm{~Hz}, \mathrm{Ar}-\mathrm{H}\right), 7.28-7.16(\mathrm{~m}, 5 \mathrm{H}, \mathrm{Ar}-\mathrm{H})$, $6.85\left(\mathrm{ddd}, 1 \mathrm{H}, J_{I}=1.0, \mathrm{~J}_{2}=J_{3}=8.2 \mathrm{~Hz}, \mathrm{Ar}-\mathrm{H}\right), 4.34\left(\mathrm{ddd}, 2 \mathrm{H}, J_{I}=5.6, J_{2}=J_{3}=7.8 \mathrm{~Hz}, \mathrm{~N}-\mathrm{CH}_{2}\right), 4.16(\mathrm{q}$, 
$\left.2 \mathrm{H}, J=7.1 \mathrm{~Hz},-\mathrm{CH}_{2}\right), 3.79\left(\mathrm{~s}, 3 \mathrm{H}, \mathrm{O}-\mathrm{CH}_{3}\right), 3.03\left(\mathrm{ddd}, 2 \mathrm{H}, J_{l}=5.8, J_{2}=J_{3}=7.4 \mathrm{~Hz}, \mathrm{Ar}-\mathrm{CH}_{2}\right), 1.30(\mathrm{t}, 3 \mathrm{H}$, $\left.J=7.2 \mathrm{~Hz},-\mathrm{CH}_{3}\right) \mathrm{ppm} ;{ }^{13} \mathrm{C}-\mathrm{NMR}: \delta 161.6,150.4,157.5,139.1,134.5,130.2,128.8,127.4,122.2$, $120.1,112.9,109.9,55.1,41.5,38.5,29.6,12.5$ ppm; ESI-MS (m/e): 324.1[M+ H $]^{+}$.

1-Ethyl-3-[2'-(3'-methoxyphenyl)ethyl]-lH,3H-quinazoline-2,4-dione (1bd). White solid. Yield >94\%; mp. $112-114^{\circ} \mathrm{C}$; IR (NaCl): 2966, 2841, 1701, 1653, 1605, 1483, $1258 \mathrm{~cm}^{-1}$; ${ }^{1} \mathrm{H}-\mathrm{NMR}: \delta 8.25$ (dd, $1 \mathrm{H}$, $\left.J_{I}=1.6, J_{2}=8.0 \mathrm{~Hz}, \mathrm{Ar}-\mathrm{H}\right), 7.68\left(\mathrm{ddd}, 1 \mathrm{H}, J_{l}=1.7, J_{2}=7.3, J_{3}=8.5 \mathrm{~Hz}, \mathrm{Ar}-\mathrm{H}\right), 7.29-7.18(\mathrm{~m}, 4 \mathrm{H}, \mathrm{Ar}-\mathrm{H})$, 6.91(m, 1H, Ar-H), $6.77\left(\mathrm{ddd}, 1 \mathrm{H}, J_{I}=0.9, J_{2}=2.6, J_{3}=8.2 \mathrm{~Hz}, \mathrm{Ar}-\mathrm{H}\right), 4.31$ (ddd, $2 \mathrm{H}, J_{l}=5.6$, $\left.\mathrm{J}_{2}=J_{3}=7.8 \mathrm{~Hz}, \mathrm{~N}-\mathrm{CH}_{2}\right), 4.20\left(\mathrm{q}, 2 \mathrm{H}, J=7.1 \mathrm{~Hz},-\mathrm{CH}_{2}\right), 3.79\left(\mathrm{~s}, 3 \mathrm{H}, \mathrm{O}-\mathrm{CH}_{3}\right), 2.97$ (ddd, $2 \mathrm{H}, J_{l}=5.6$, $\left.J_{2}=J_{3}=8.4 \mathrm{~Hz}, \mathrm{Ar}-\mathrm{CH}_{2}\right), 1.35\left(\mathrm{t}, 3 \mathrm{H}, J=7.2 \mathrm{~Hz},-\mathrm{CH}_{3}\right)$ ppm; ${ }^{13} \mathrm{C}-\mathrm{NMR}: \delta$ 161.6, 150.7, 140.2, 135.0, $131.5,129.4,129.1,122.7,121.4,114.2,113.3,112.3,55.1,43.1,38.7,34.0,12.5$ ppm; ESI-MS (m/e): $324.1[\mathrm{M}+\mathrm{H}]^{+}$.

1-Ethyl-3-[2'-(4'-methoxyphenyl)ethyl]-lH,3H-quinazoline-2,4-dione (1be). White solid. Yield >97\%; mp.110-112 ${ }^{\circ}$; IR (NaCl): 2974, 2833, 1701, 1657, 1609, 1509, 1483, $1244 \mathrm{~cm}^{-1} ;{ }^{1} \mathrm{H}-\mathrm{NMR}: \delta 8.24$ $\left(\mathrm{dd}, 1 \mathrm{H}, J_{I}=1.7, J_{2}=7.8 \mathrm{~Hz}, \mathrm{Ar}-\mathrm{H}\right), 7.66$ (ddd, $\left.1 \mathrm{H}, J_{I}=1.4, J_{2}=J_{3}=8.4 \mathrm{~Hz}, \mathrm{Ar}-\mathrm{H}\right), 7.28-7.18$ (m, $4 \mathrm{H}, \mathrm{Ar}-$ $\mathrm{H}), 6.84\left(\mathrm{ddd}, 2 \mathrm{H}, J_{I}=2.2, J_{2}=J_{3}=6.6 \mathrm{~Hz}, \mathrm{Ar}-\mathrm{H}\right), 4.31-4.12\left(\mathrm{~m}, 4 \mathrm{H}, 2 \mathrm{~N}-\mathrm{CH}_{2}\right), 3.77$ (s, 3H, O-CH$)$, $2.93\left(\mathrm{ddd}, 2 \mathrm{H}, J_{I}=5.6, J_{2}=J_{3}=8.2 \mathrm{~Hz}, \mathrm{Ar}-\mathrm{CH}_{2}\right), 1.34\left(\mathrm{t}, 3 \mathrm{H}, J=7.1 \mathrm{~Hz},-\mathrm{CH}_{3}\right) \mathrm{ppm} ;{ }^{13} \mathrm{C}-\mathrm{NMR}: \delta 161.6$, 158., 150.3, 139.5, 135.0, 130.7, 129.9, 129.1, 122.7, 115.7, 113.9, 113.3, 55.2, 43.3, 38.7, 33.0, 12.5 ppm; ESI-MS (m/e): 324.1[M+ H] .

1-Ethyl-3-(3-(4-phenylpiperazin-1-yl)propyl)-1H,3H-quinazoline-2,4-dione (1bf). White solid. Yield $>92 \%$; mp. 94-96 ${ }^{\circ} \mathrm{C}$; IR (KBr): 3010, 2937, 2880, 2803, 1695, 1647, 1604, 1223, $1110 \mathrm{~cm}^{-1} .{ }^{1} \mathrm{H}-\mathrm{NMR}$ : $\delta 8.10\left(\mathrm{dd}, 1 \mathrm{H}, J_{I}=1.6, J_{2}=7.8 \mathrm{~Hz}, \mathrm{Ar}-\mathrm{H}\right), 7.73\left(\mathrm{ddd}, 1 \mathrm{H}, J_{I}=1.8, J_{2}=7.4, J_{3}=8.8 \mathrm{~Hz}, \mathrm{Ar}-\mathrm{H}\right), 7.45(\mathrm{~d}, 1 \mathrm{H}$, $J=8.4 \mathrm{~Hz}, \mathrm{Ar}-\mathrm{H}), 7.31-7.14$ (m, 3H, Ar-H), 6.89-6.72 (m, 3H, Ar-H), 4.22-4.04 (m, 4H, 2 N-CH $)_{2}$, $3.77(\mathrm{~s}, 1 \mathrm{H}), 3.19-2.99\left(\mathrm{~m}, 4 \mathrm{H}, \mathrm{N}-\mathrm{CH}_{2}\right), 2.56-2.40\left(\mathrm{~m}, 6 \mathrm{H}, \mathrm{N}-\mathrm{CH}_{2}\right), 1.86\left(\mathrm{dd}, 3 \mathrm{H}, J_{I}=7.0, J_{2}=14.2 \mathrm{~Hz}\right.$, $\mathrm{CH}_{2}$ ), $1.25\left(\mathrm{t}, 3 \mathrm{H}, J=7.1 \mathrm{~Hz}, \mathrm{CH}_{3}\right)$ ppm; ${ }^{13} \mathrm{C}-\mathrm{NMR}: \delta 164.9,154.7,153.7,143.1,138.8,132.5,131.8$, $126.2,122.6,119.1,118.9,117.7,59.4,56.5,52.1,51.4,43.32,28.0,16.0$ ppm; ESI-MS (m/e): $392.2[\mathrm{M}+\mathrm{H}]^{+}$.

\section{Acknowledgements}

We are grateful to Consejo Nacional de Ciencia y Tecnología in México (CONACYT, grant No. SEP-2004-CO1-47835) and Dirección General de Educación Superior Tecnológica (DGEST) for supporting this project. Leticia Guerrero thanks to CONACYT for a scholarship.

\section{References}

1. Piers, E.; Grierson, R.J. Alkylation of 1,5-dimethoxy-1,4-cyclohexadiene. A convenient synthesis of 2-alkyl-and-2-alkenyl-1,3-cyclohexanediones. J. Org. Chem. 1977, 42, 3755-3756.

2. Merz, A. Phase-transfer-catalyzed Alkylation of Alcoholes by Dimethyl Sulfate in an Aqueous System. Angew. Chem. Int. 2003, 12, 846-847.

3. Haniti, M.; Hamid, S.A.; Williams, J.M.J. Ruthenium catalyzed $N$-alkylation of amines with alcohols. Chem. Commun. 2007, 725- 727. 
4. MacPhee, J.A.; Dubois, J.E. Steric effects in synthesis-steric limits to the alkylation of nitriles and carboxylic acids. Tetrahedron 1980, 36, 775-777.

5. Goto, S.; Tsuboi, H.; Kanoda, M.; Mukai, K.; Kagara, K. The Process Development of a Novel Aldose Reductase Inhibitor, FK366. Part 1. Improvement of Discovery Process and New Synthesis of 1-Substituted Quinazolinediones. Org. Process Res. Dev.2003, 7, 700-706.

6. Larksarp, C.; Alper, H. Palladium-Catalyzed Cyclocarbonylation of $O$-Iodoanilines with Heterocumulenes: Regioselective Preparation of 4(3H)-Quinazoline Derivatives. J. Org. Chem . 2000, 65, 2773-2777.

7. Hermecz, I.; Kökosi, J. Podanyi, B.; Szasz, G. Synthesis of Indolyl-4(3H)-Quinazolinones. Heterocycles 1994, 37, 903-914.

8. Katritzky, A.R.; Rees, C.W. Comprehensive Heterocyclic Chemis try: The Structure, Reactions, Synthesis and Uses of He terocyclic Compounds, Part 2B ; Pergamon Press: New York, USA, 1984; Volume 3.

9. Pelletier, S.W. Alkaloids: Chemical and Biological Perspectives ; John Wiley \& Sons Ltd.: New York, USA, 1985; Volume 1.

10. Scovill, J.; Blank, E.; Konnick, M.; Nenortas, E.; Shapiro, T. Antitrypanosomal Activities of Tryptanthrins. Antimicrob. Agents Chemoter. 2002, 46, 882-883.

11. Penn, J.; Mantle, P.G.; Bilton, J.N.; Sheppard, R.N. Glyantrypine, a novel anthranilic acidcontaining metabolite of Aspergillus clavatus. J. Chem. Soc. Perkin Trans. 1, 1992, 1495-1496.

12. Wong, S-M.; Musza, L.L.; Kydd, G.C.; Kullnig, R.; Gillum, A.M.; Cooper. R. Fiscalins: new substance $\mathrm{P}$ inhibitors produced by the fungus Neosartorya fischeri. J. Antibiot. 1993, 46, 545553.

13. Fujimoto, H.; Negishi, E.; Yamaguchi, K.; Nishi, N.; Yamazaki, M. Isolation of new tremorgenic metabolites from an ascomycete, Corynascus setosus. Chem. Pharm. Bull. 1996, 44, 1843-1848.

14. Numata, A.; Takahashi, C.; Matsushita, T.; Miyamoto, T.; Kawai, K.; Usami. Y.; Matsumura, E.; Inoue, M.; Ohishi, H.; Shingu, T. Fumiquinazolines, novel metabolites of a fungus isolated from a saltfish. Tetrahedron Lett. 1992, 33, 1621-1624.

15. Takahashi, C.; Matsushita, T.; Doi, M.; Minoura, K.; Shingu, T.; Kumeda, Y.; Numata, A. Fumiquinazolines A-G, novel metabolites of a fungus separated from a Pseudolabrus marine fish. J. Chem. Soc. Perkin Trans 1 1995, 2345-2353.

16. Karwowski, J. P.; Jackson, M.; Rasmussen, R. R.; Humphrey, P. E.; Poddig, J. B.; Kohl, W. L.; Scherr, M.H.; Kadam, S.; McAlpine, J.B. 5-N-Acetylardeemin, a novel heterocyclic compound which reverses multiple drug resistance in tumor cells. J. Antibiot.1993, 46, 374-379.

17. Hochlowski, J.E.; Mullally, M.M.; Spanton, S.G.; Whittern, D.N.; Hill, P.; McAlpine. J.B. 5-NAcetylardeemin, a novel heterocyclic compound which reverses multiple drug resistance in tumor cells. J. Antibiot. 1993, 46, 380-386.

18. Larsen, T.O.; Frydenvang, K.; Frisvad, J.C.; Christophersen, C. UV-Guided Isolation of Alantrypinone, a Novel Penicillium Alkaloid. J. Nat. Prod. 1998, 61, 1154-1157.

19. Barrow, C. J.; Sun, H. H. Spiroquinazoline, a Novel Substance P Inhibitor with a New Carbon Skeleton, Isolated from Aspergillus flavipes. J. Nat. Prod. 1994, 57, 471-476. 
20. Hernández, F.; Buenadicha, F.L.; Avendaño, C.; Söllhuber, M. 1-Alkyl-2,4-dihydro-1Hpyrazino[2,1-b]quinazoline-3,6-diones as glycine templates. Synthesis of Fiscalin B. Tetrahedron Asymmetry 2001, 12, 3387-3398.

21. Hayao, S.; Havera, H.J.; Strycker, W.G.; Hong, E. Hypotensive, antiadrenergic, and antihistaminic 3-substituded 2-methyl-(or 2-phenyl-)4(3H)-quinazolones. J. Med. Chem . 1969, 12(5), 936-938.

22. Shiau, C.Y.; Chern, J.W.; Tien, J.H.; Liu, K C. Reactions of 2-Aminothiobenzamide with Isocyanates: A New Synthesis of 2,3-Dihydroimidazo[1,2-c]quinazolin-5(6H)-one and 3,4Dihydro-2H-pyrimido[1,2-c]quinazolin-6(7H)-one. J. Heterocyclic Chem. 1989, 26, 595-596.

23. Nishikawa, Y.; Shindo, T.; Ishii, K.; Nakamura, H.; Kon, T.; Uno, H. Acrylamide derivatives as antiallergic agents. 2. Synthesis and structure activity relationships of N-[4-[4-(diphenylmethyl)1-piperazinyl]butyl]-3-(3-pyridyl)acrylamides. J. Med. Chem. 1989, 32, 583-593.

24. Hayao, S.; Havera, H.J.; Strycker, W.G.; Leipzig, T.J.; Kulp, R.A.; Hartzler, H.E. New Sedative and Hypotensive 3-Substituted 2,4(1H,3H)-Quinazolinediones. J. Med. Chem. 1965, 8, 807-811.

25. Hayao, S. Quinazolinedione derivatives. US Patent 3274194, 1965.

26. Cortez, R.; Rivero, I.A.; Somanathan R.; Aguirre G.; Ramirez , F.; Hong, E. Synthesis of Quinazolinedione Using Triphosgene. Synth. Commun. 1991, 21, 285-292.

27. Rivero, I.A.; Somanathan, R.; Hellberg, L.H. Synthesis of 3-Dipeptidyl-2,4(1H,3H)Quinazolinediones as Potential Anti-hypertensive Agents. Synth. Commun. 1998, 28, 2077-2086.

28. Dreyer, D.L.; Brenner, R.C. Alkaloids of some Mexican Zanthoxylum species. Phytochemistry 1980, 19, 935-939.

29. Rivero, I.A.; Espinoza, K.; Somanathan, R. Synthesis of Quinazoline-2,4-dione Alkaloids and Analogues from Mexican Zanthoxylum Species. Molecules 2004, 9, 609-616.

30. Hunig, S.; Quast, H.; Brenninger, W.; Frankenfield, E. Tetramethyl-p-phenylenediamine. Org. Synth. 1973, 5, 1018-1021.

Sample Availability: Available from the authors.

(C) 2009 by the authors; licensee Molecular Diversity Preservation International, Basel, Switzerland. This article is an open-access article distributed under the terms and conditions of the Creative Commons Attribution license (http://creativecommons.org/licenses/by/3.0/). 